\title{
MULTIFACTORIAL TESTING OF ROLLER BEARING RELIABILITY
}

\section{Živan Arsenić}

University of Belgrade, Faculty of Mechanical Engineering, Kraljice Marije 16, 11000 Belgrade, Serbia

\section{() MESTE NGO}

JEL category: C0, C02, C6, C63

\section{Summary:}

This paper describes one model of the method of accelerated multi-factorial testing of roller bearing reliability, that represents the result of many years of work and research of the author in the field of technical system effectiveness, and that treats, from the theoretical point of view, a completely new method of multi-factorial reliability testing, which the broader scientific and expert circles are not yet familiar with. The method of accelerated multi-factorial testing of roller bearing reliability, treated by this work, contains significant advantages with respect to all other methods of reliability testing, which have been used so far in the engineering reliability testing practice in the world.

\section{Keywords:}

reliability, multifactorial testing, roller bearing, effectiveness

\section{Introduction}

The basic attributes of the method of accelerated multi-factorial testing of reliability of a mechanical system elements, described in this work, are as described in the text below:

- The function of the diagnostical parameter change in time is attained within the desired scope of the parameter;

- New plans for reliability testing in multifactorial area (space).

- New statistical method based on regression

The address of the author:

Živan Arsenić

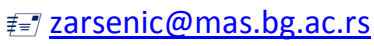

analysis.

- Enormous decrease of the number of experiments, and thereby of the costs of reliability testing in multi-factorial space.

- Interpretation of testing results is enabled in the form of Weibull distribution law in the whole defined multi-factorial space.

\section{Influential factors}

Testing of roller bearing reliability in this example is done in the seven-factor space, where the influential factors are given in the Table 1 . 


\section{Table 1. Influential factors}

\begin{tabular}{|c|c|c|c|c|c|c|}
\hline Factors & Factor Type & Abbreviation & $\begin{array}{c}\text { Unit of } \\
\text { Measure }\end{array}$ & $\begin{array}{c}\text { Bottom } \\
\text { Level } \\
\mathbf{X}_{\mathbf{d}}\end{array}$ & $\begin{array}{c}\text { Top } \\
\text { Level } \\
\mathbf{X}_{\mathbf{g}}\end{array}$ & $\begin{array}{c}\text { Variation } \\
\text { Interval } \\
\mathbf{W}=\left(\mathbf{X}_{\mathbf{g}} \mathbf{-} \mathbf{X}_{\mathbf{d}} \mathbf{)} \mathbf{2}\right.\end{array}$ \\
\hline $\mathrm{X}_{1}$ & $\begin{array}{c}\text { Mean value of radial } \\
\text { loadnormal } \\
\text { distribution }\end{array}$ & $\mathrm{M}_{\mathrm{r}}$ & $\mathrm{kN}$ & 2 & 4 & 1 \\
\hline $\mathrm{X}_{2}$ & $\begin{array}{c}\text { Standard deviation } \\
\text { of radial loadnormal } \\
\text { distribution }\end{array}$ & $\sigma_{\mathrm{r}}$ & $\mathrm{kN}$ & 0.5 & 2 & 0.75 \\
\hline $\mathrm{X}_{3}$ & $\begin{array}{c}\text { Mean value of axial } \\
\text { loadnormal } \\
\text { distribution }\end{array}$ & $\mathrm{M}_{\mathrm{a}}$ & $\mathrm{kN}$ & 0 & 2 & 1 \\
\hline $\mathrm{X}_{4}$ & $\begin{array}{c}\text { Standard deviation } \\
\text { of axial loadnormal } \\
\text { distribution }\end{array}$ & $\sigma_{\mathrm{a}}$ & $\mathrm{kN}$ & 0.5 & 2 & 0.75 \\
\hline $\mathrm{X}_{5}$ & Viscosity & $\mathrm{k}$ & & 0.1 & 4 & 1.95 \\
\hline $\mathrm{X}_{6}$ & Contamination & $\eta_{\mathrm{c}}$ & & 0 & 1 & 0.5 \\
\hline $\mathrm{X}_{7}$ & $\begin{array}{c}\text { Diagnostic } \\
\text { parameter }\end{array}$ & $\mathrm{D}$ & $\mathrm{mm}$ & 3 & 6 & 1.5 \\
\hline
\end{tabular}

\section{Testing plan}

The plan-testing matrix is formed in the following way:

The reliability testing plan matrix in a sevenfactor area is arrived at, by taking the sevenfactor process as the initial basis, whose complete orthogonal plan $2^{7}$ contains 128 different points. The $2^{7-3}=2^{4}$ replica, with the number of 16 experiments, is formed by choosing of the next generator, i.e. the corresponding contrast (Koldžić, 1999), (Stanić, 1990)::

$$
\begin{array}{ll}
x_{5}=x_{1} x_{2} & J=x_{1} x_{2} x_{5} \\
x_{6}=x_{3} x_{4} & J=x_{3} x_{4} x_{6} \\
x_{7}=x_{1} x_{4} & J=x_{1} x_{4} x_{7}
\end{array}
$$

$J=x_{1} x_{2} x_{5}=x_{3} x_{4} x_{6}=x_{1} x_{4} x_{7}=x_{2} x_{4} x_{5} x_{7}=x_{1} x_{3} x_{6} x_{7}$

The testing plan and the results are given in the Table 2, where $x_{1}, x_{2}, x_{3}, x_{4}, x_{5}, x_{6}, x_{7}$, are the coded values of the factors $X_{1}, X_{2}, X_{3}, X_{4}$, $x_{5}, X_{6}, x_{7}$, so that 1 in the Table 2 represents the maximum factor value (the top level in the Table 1), with $\mathbf{- 1}$ representing the minimum value (bottom level in the Table 1). The experiments are conducted, obviously, only at maximum and minimum values of influential factors. The experimental results are given in millions of $(r)$ revolutions of roller bearings to failure. 
Table 2 The testing plan and results

\begin{tabular}{|c|c|c|c|c|c|c|c|c|c|c|c|c|}
\hline \multirow{3}{*}{ 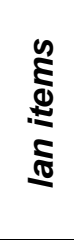 } & \multirow{2}{*}{\multicolumn{7}{|c|}{ Plan matrix $X s$}} & \multicolumn{5}{|c|}{ Experimental results $Y$} \\
\hline & & & & & & & & \multicolumn{5}{|c|}{${ }^{*} y=10^{-1} \ln r, \quad \bar{y}=\Sigma y / 4$} \\
\hline & $x_{1}$ & $x_{2}$ & $x_{3}$ & $x_{4}$ & $x_{5}$ & $x_{6}$ & $x_{7}$ & $y_{1}$ & $y_{2}$ & $y_{3}$ & $y_{4}$ & $\bar{y}^{-}$ \\
\hline 1 & 1 & 1 & 1 & 1 & 1 & 1 & 1 & 0.7 & 1.4 & 2.1 & 2.3 & 1.625 \\
\hline 2 & 1 & 1 & 1 & -1 & 1 & -1 & -1 & 0.05 & 1 & 1.4 & 2.2 & 1.1125 \\
\hline 3 & 1 & 1 & -1 & 1 & 1 & -1 & 1 & 0.9 & 1.4 & 2.2 & 2.8 & 1.825 \\
\hline 4 & 1 & 1 & -1 & -1 & 1 & 1 & -1 & 0.9 & 1.3 & 2 & 2.8 & 1.975 \\
\hline 5 & 1 & -1 & 1 & 1 & -1 & 1 & 1 & 1.2 & 2 & 2.3 & 3 & 2.125 \\
\hline 6 & 1 & -1 & 1 & -1 & -1 & -1 & -1 & 1.3 & 2 & 2.6 & 3.2 & 2.275 \\
\hline 7 & 1 & -1 & -1 & 1 & -1 & -1 & 1 & 1.3 & 2.1 & 2.7 & 3.1 & 2.325 \\
\hline 8 & 1 & -1 & -1 & -1 & -1 & 1 & -1 & 5.4 & 6 & 6.8 & 7.4 & 6.4 \\
\hline 9 & -1 & 1 & 1 & 1 & -1 & 1 & -1 & 1.5 & 2.2 & 2.5 & 3.4 & 2.4 \\
\hline 10 & -1 & 1 & 1 & -1 & -1 & -1 & 1 & 2 & 2.5 & 3 & 3.9 & 2.85 \\
\hline 11 & -1 & 1 & -1 & 1 & -1 & -1 & -1 & 2.1 & 3 & 3.8 & 4.3 & 3.3 \\
\hline 12 & -1 & 1 & -1 & -1 & -1 & 1 & 1 & 6.4 & 7 & 7.6 & 8.3 & 7.325 \\
\hline 13 & -1 & -1 & 1 & 1 & 1 & 1 & -1 & 3 & 3.5 & 4.3 & 4.9 & 3.9 \\
\hline 14 & -1 & -1 & 1 & -1 & 1 & -1 & 1 & 4 & 4.6 & 5.5 & 5.8 & 4.975 \\
\hline 15 & -1 & -1 & -1 & 1 & 1 & -1 & -1 & 1 & 1.3 & 2 & 2.8 & 1.77 \\
\hline 16 & -1 & -1 & -1 & -1 & 1 & 1 & 1 & 7 & 8 & 8.4 & 9 & $8.1^{-}$ \\
\hline
\end{tabular}

$* r-$ number of revolution till failure

\section{Testing results processing}

\begin{tabular}{|c|c|c|c|c|c|c|c|c|c|c|c|c|c|c|c|}
\hline & $x_{0}$ & $x_{1}$ & $x_{2}$ & $x_{3}$ & $x_{4}$ & $x_{5}$ & $x_{6}$ & $x_{7}$ & $x_{2} x_{4}$ & $x_{2} x_{3}$ & $x_{1} x_{6}$ & $x_{2} x_{6}$ & $x_{4} x_{5}$ & $x_{3} x_{5}$ & $x_{5} x_{6}$ \\
\hline & 1 & 1 & 1 & 1 & 1 & 1 & 1 & 1 & 1 & 1 & 1 & 1 & 1 & 1 & 1 \\
\hline & 1 & 1 & 1 & 1 & -1 & 1 & -1 & -1 & -1 & 1 & -1 & -1 & -1 & 1 & -1 \\
\hline & 1 & 1 & 1 & -1 & 1 & 1 & -1 & 1 & 1 & -1 & -1 & -1 & 1 & -1 & -1 \\
\hline & 1 & 1 & 1 & -1 & -1 & 1 & 1 & -1 & -1 & -1 & 1 & 1 & -1 & -1 & 1 \\
\hline & 1 & 1 & -1 & 1 & 1 & -1 & 1 & 1 & -1 & -1 & 1 & -1 & -1 & -1 & -1 \\
\hline & 1 & 1 & -1 & 1 & -1 & -1 & -1 & -1 & 1 & -1 & -1 & 1 & 1 & -1 & 1 \\
\hline & 1 & 1 & -1 & -1 & 1 & -1 & -1 & 1 & -1 & 1 & -1 & 1 & -1 & 1 & 1 \\
\hline$\Lambda_{S}-$ & 1 & 1 & -1 & -1 & -1 & -1 & 1 & -1 & 1 & 1 & 1 & -1 & 1 & 1 & -1 \\
\hline & 1 & -1 & 1 & 1 & 1 & -1 & 1 & -1 & 1 & 1 & -1 & 1 & -1 & -1 & -1 \\
\hline & 1 & -1 & 1 & 1 & -1 & -1 & -1 & 1 & -1 & 1 & 1 & -1 & 1 & -1 & 1 \\
\hline & 1 & -1 & 1 & -1 & 1 & -1 & -1 & -1 & 1 & -1 & 1 & -1 & -1 & 1 & 1 \\
\hline & 1 & -1 & 1 & -1 & -1 & -1 & 1 & 1 & -1 & -1 & -1 & 1 & 1 & 1 & -1 \\
\hline & 1 & -1 & -1 & 1 & 1 & 1 & 1 & -1 & -1 & -1 & -1 & -1 & 1 & 1 & 1 \\
\hline & 1 & -1 & -1 & 1 & -1 & 1 & -1 & 1 & 1 & -1 & 1 & 1 & -1 & 1 & -1 \\
\hline & 1 & -1 & -1 & -1 & 1 & 1 & -1 & -1 & -1 & 1 & 1 & 1 & 1 & -1 & -1 \\
\hline & 1 & -1 & -1 & -1 & -1 & 1 & 1 & 1 & 1 & 1 & -1 & -1 & -1 & -1 & 1 \\
\hline
\end{tabular}




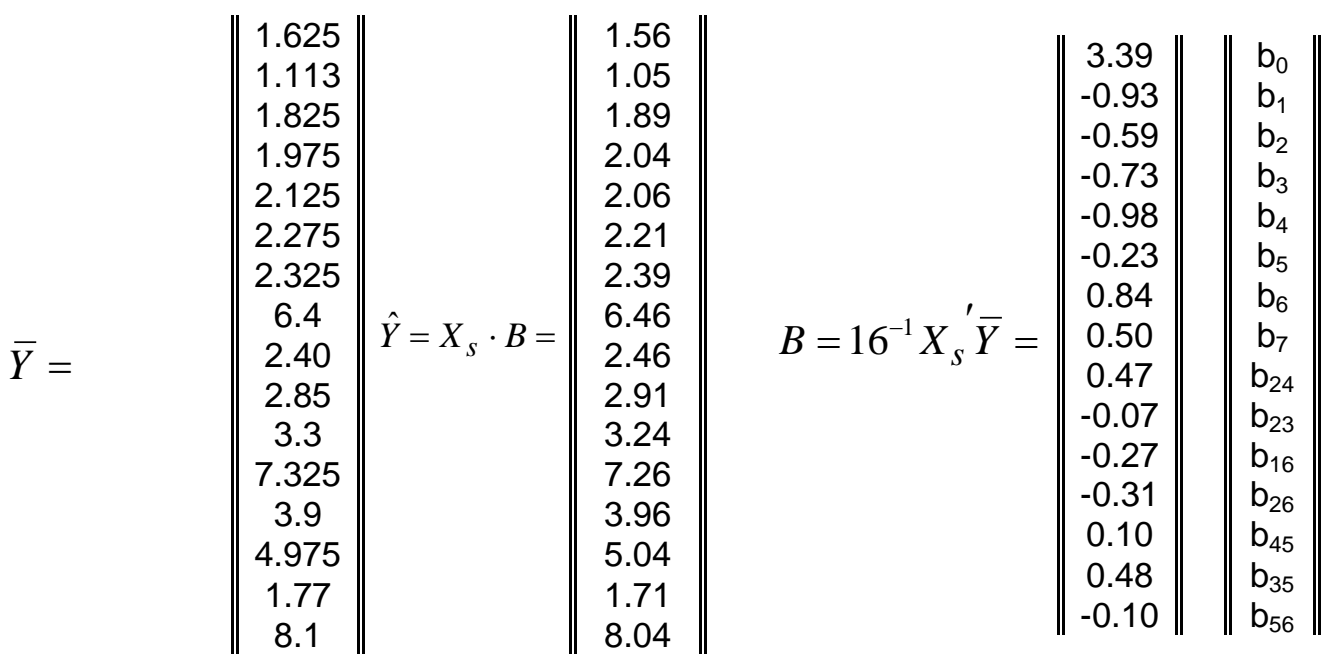

\section{Table 3 The testing results}

\begin{tabular}{|c|c|c|c|c|c|c|c|c|c|c|}
\hline \multicolumn{4}{|c|}{$\begin{aligned} e_{1} & =y_{1}-\hat{y} \\
e_{2} & =y_{2}-\hat{y} \\
e_{3} & =y_{3}-\hat{y} \\
e_{4} & =y_{4}-\hat{y} \\
e & =\bar{y}-\hat{y}\end{aligned}$} & \multicolumn{3}{|c|}{$\begin{aligned} m & =\frac{\bar{e}}{|\bar{e}|} \\
m & =\frac{\bar{e}_{1}}{\bar{e}_{j}}\end{aligned}$} & \multicolumn{4}{|c|}{$\begin{array}{c}\boldsymbol{E} \\
E_{1}=e_{1} m \\
E_{2}=e_{2} m \\
E_{3}=e_{3} m \\
E_{4}=e_{4} m\end{array}$} \\
\hline$\hat{y}$ & $\bar{e}$ & $e_{1}$ & $e_{2}$ & $e_{3}$ & $e_{4}$ & $m$ & $E_{1}$ & $E_{2}$ & $E_{3}$ & $E_{4}$ \\
\hline 1.564 & 0.061 & -0.86 & -0.16 & 0.54 & 0.74 & 1 & -0.86 & -0.16 & 0.54 & 0.74 \\
\hline 1.051 & 0.061 & -1.00 & -0.05 & 0.35 & 1.15 & 1 & -1.00 & -0.05 & 0.35 & 1.15 \\
\hline 1.886 & -0.061 & -0.99 & -0.49 & 0.31 & 0.91 & -1 & 0.99 & 0.49 & -0.31 & -0.91 \\
\hline 2.036 & -0.061 & -1.14 & -0.74 & -0.04 & 0.76 & -1 & 1.14 & 0.74 & 0.04 & -0.76 \\
\hline 2.064 & 0.061 & -0.86 & -0.06 & 0.24 & 0.94 & 1 & -0.86 & -0.06 & 0.24 & 0.94 \\
\hline 2.214 & 0.061 & -0.91 & -0.21 & 0.39 & 0.99 & 1 & -0.91 & -0.21 & 0.39 & 0.99 \\
\hline 2.386 & -0.061 & -1.09 & -0.29 & 0.31 & 0.71 & -1 & 1.09 & 0.29 & -0.31 & -0.71 \\
\hline 6.461 & -0.061 & -1.06 & -0.46 & 0.34 & 0.94 & -1 & 1.06 & 0.46 & -0.34 & -0.94 \\
\hline 2.461 & -0.061 & -0.96 & -0.26 & 0.04 & 0.94 & -1 & 0.96 & 0.26 & -0.04 & -0.94 \\
\hline 2.911 & -0.061 & -0.91 & -0.41 & 0.09 & 0.99 & -1 & 0.91 & 0.41 & -0.09 & -0.99 \\
\hline 3.239 & 0.061 & -1.14 & -0.24 & 0.56 & 1.06 & 1 & -1.14 & -0.24 & 0.56 & 1.06 \\
\hline 7.264 & 0.061 & -0.86 & -0.26 & 0.34 & 1.04 & 1 & -0.86 & -0.26 & 0.34 & 1.04 \\
\hline 3.961 & -0.061 & -0.96 & -0.46 & 0.34 & 0.94 & -1 & 0.96 & 0.46 & -0.34 & -0.94 \\
\hline 5.036 & -0.061 & -1.04 & -0.44 & 0.46 & 0.76 & -1 & 1.04 & 0.44 & -0.46 & -0.76 \\
\hline 1.709 & 0.061 & -0.71 & -0.41 & 0.29 & 1.09 & 1 & -0.71 & -0.41 & 0.29 & 1.09 \\
\hline 8.039 & 0.061 & -1.04 & -0.04 & 0.36 & 0.96 & 1 & -1.04 & -0.04 & 0.36 & 0.96 \\
\hline
\end{tabular}




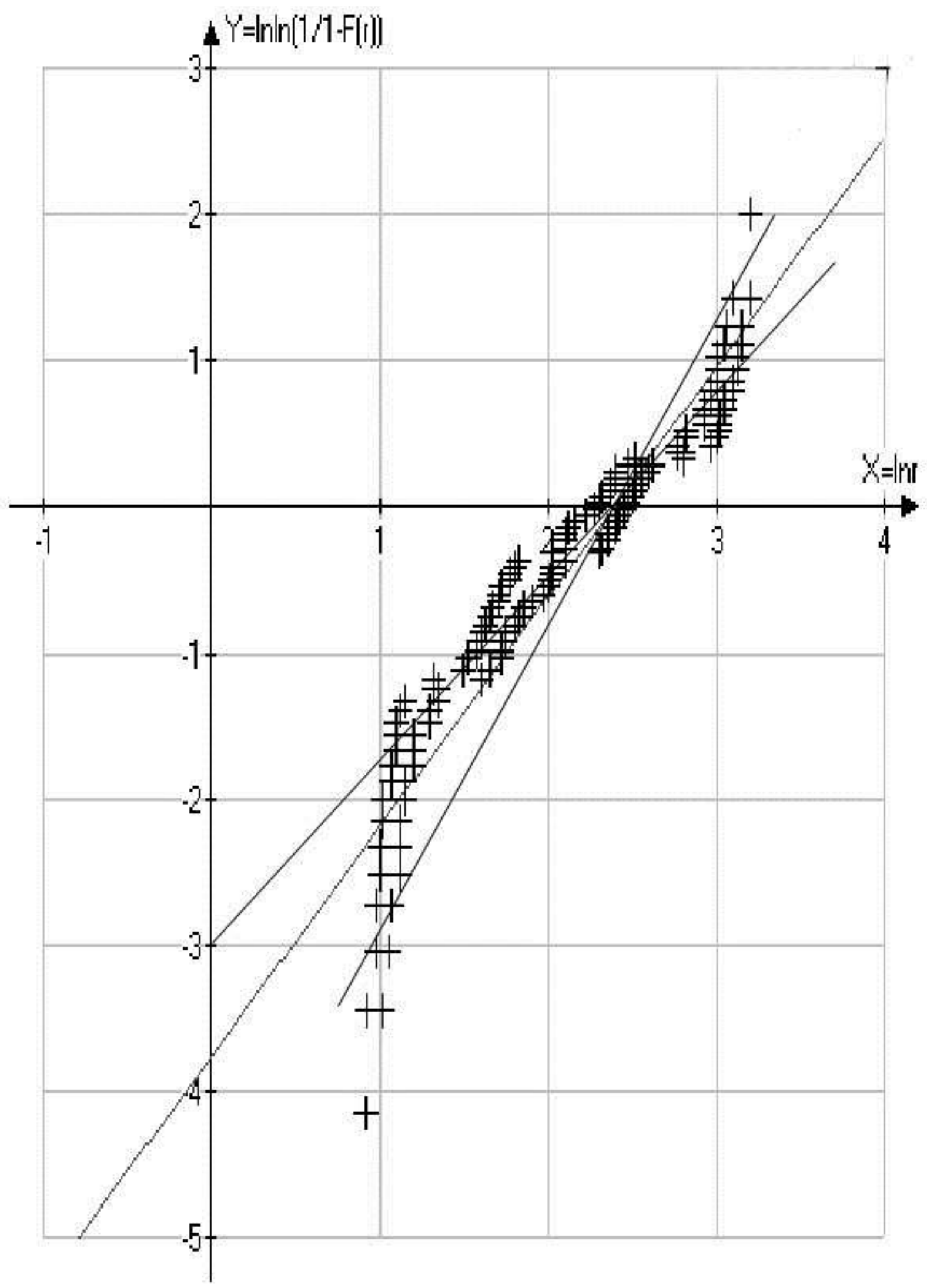

Figure 1 The testing results 


\begin{tabular}{|c|c|c|c|c|c|c|c|c|c|c|c|c|c|}
\hline $\begin{array}{l}{ }^{*} \text { The } \\
\text { anot }\end{array}$ & $\begin{array}{l}\overline{e m} \\
\text { er o }\end{array}$ & $\begin{array}{l}\text { ler } \\
\text { by t }\end{array}$ & $\begin{array}{l}\mathrm{s} \text { lin } \\
\mathrm{dec}\end{array}$ & $\begin{array}{l}\text { in } \\
\text { ing }\end{array}$ & $X$ & $\begin{array}{l}\text { רn by } \\
5+E\end{array}$ & rising & $y 5$ & & $Y=\ln$ & $n(1-n)$ & $\overline{(1-n)}$ & $N=64$ \\
\hline$n$ & ${ }^{*} E$ & ${ }^{*} E$ & $\hat{y}_{5}$ & $x^{\prime}$ & $x^{\prime \prime}$ & $1-n / N$ & $(1-n / M)^{-1}$ & $Y$ & $Y$ & $\left(X^{\prime}\right)^{2}$ & $\left(X^{\prime \prime}\right)^{2}$ & $X^{\prime} \cdot Y$ & $X^{\prime \prime} \cdot Y$ \\
\hline 1 & -1.14 & 1.15 & 2.06 & 0.92 & 0.91 & 0.98 & 1.02 & 0.02 & -4.15 & 0.85 & 0.83 & -3.82 & -3.78 \\
\hline 2 & -1.04 & 1.14 & 2.06 & 1.02 & 0.92 & 0.97 & 1.03 & 0.03 & -3.45 & 1.04 & 0.85 & -3.52 & -3.19 \\
\hline 3 & -1.00 & 1.09 & 2.06 & 1.06 & 0.97 & 0.95 & 1.05 & 0.05 & -3.04 & 1.12 & 0.94 & -3.22 & -2.94 \\
\hline 4 & -0.99 & 1.09 & 2.06 & 1.07 & 0.97 & 0.94 & 1.07 & 0.06 & -2.74 & 1.15 & 0.95 & -2.94 & -2.67 \\
\hline 5 & -0.94 & 1.06 & 2.06 & 1.12 & 1.00 & 0.92 & 1.08 & 0.08 & -2.51 & 1.26 & 1.00 & -2.81 & -2.51 \\
\hline 6 & -0.94 & 1.06 & 2.06 & 1.12 & 1.00 & 0.91 & 1.10 & 0.10 & -2.32 & 1.26 & 1.00 & -2.60 & -2.32 \\
\hline 7 & -0.94 & 1.04 & 2.06 & 1.12 & 1.02 & 0.89 & 1.12 & 0.12 & -2.16 & 1.26 & 1.05 & -2.42 & -2.21 \\
\hline 8 & -0.91 & 1.04 & 2.06 & 1.15 & 1.02 & 0.88 & 1.14 & 0.13 & -2.01 & 1.31 & 1.05 & -2.31 & -2.06 \\
\hline 9 & -0.91 & 0.99 & 2.06 & 1.15 & 1.07 & 0.86 & 1.16 & 0.15 & -1.89 & 1.31 & 1.15 & -2.16 & -2.03 \\
\hline 10 & -0.86 & 0.99 & 2.06 & 1.20 & 1.07 & 0.84 & 1.19 & 0.17 & -1.77 & 1.43 & 1.15 & -2.12 & -1.90 \\
\hline 11 & -0.86 & 0.96 & 2.06 & 1.20 & 1.10 & 0.83 & 1.21 & 0.19 & -1.67 & 1.43 & 1.21 & -2.00 & -1.83 \\
\hline 12 & -0.86 & 0.96 & 2.06 & 1.20 & 1.10 & 0.81 & 1.23 & 0.21 & -1.57 & 1.43 & 1.21 & -1.88 & -1.73 \\
\hline 13 & -0.76 & 0.96 & 2.06 & 1.30 & 1.10 & 0.80 & 1.25 & 0.23 & -1.48 & 1.68 & 1.21 & -1.92 & -1.63 \\
\hline 14 & -0.76 & 0.94 & 2.06 & 1.30 & 1.12 & 0.78 & 1.28 & 0.25 & -1.40 & 1.68 & 1.26 & -1.81 & -1.57 \\
\hline 15 & -0.71 & 0.91 & 2.06 & 1.35 & 1.15 & 0.77 & 1.31 & 0.27 & -1.32 & 1.81 & 1.32 & -1.78 & -1.52 \\
\hline 16 & -0.71 & 0.74 & 2.06 & 1.35 & 1.32 & 0.75 & 1.33 & 0.29 & -1.25 & 1.83 & 1.75 & -1.68 & -1.65 \\
\hline 17 & -0.46 & 0.74 & 2.06 & 1.60 & 1.32 & 0.73 & 1.36 & 0.31 & -1.18 & 2.55 & 1.75 & -1.88 & -1.56 \\
\hline 18 & -0.41 & 0.56 & 2.06 & 1.65 & 1.50 & 0.72 & 1.39 & 0.33 & -1.11 & 2.73 & 2.25 & -1.83 & -1.66 \\
\hline 19 & -0.34 & 0.54 & 2.06 & 1.72 & 1.52 & 0.70 & 1.42 & 0.35 & -1.04 & 2.96 & 2.32 & -1.80 & -1.59 \\
\hline 20 & -0.34 & 0.49 & 2.06 & 1.72 & 1.57 & 0.69 & 1.45 & 0.37 & -0.98 & 2.96 & 2.48 & -1.69 & -1.54 \\
\hline 21 & -0.31 & 0.46 & 2.06 & 1.75 & 1.60 & 0.67 & 1.49 & 0.40 & -0.92 & 3.05 & 2.56 & -1.61 & -1.47 \\
\hline 22 & -0.31 & 0.46 & 2.06 & 1.75 & 1.60 & 0.66 & 1.52 & 0.42 & -0.86 & 3.05 & 2.56 & -1.51 & -1.38 \\
\hline 23 & -0.26 & 0.44 & 2.06 & 1.80 & 1.62 & 0.64 & 1.56 & 0.45 & -0.81 & 3.23 & 2.64 & -1.45 & -1.31 \\
\hline 24 & -0.24 & 0.41 & 2.06 & 1.82 & 1.65 & 0.63 & 1.60 & 0.47 & -0.76 & 3.32 & 2.72 & -1.38 & -1.24 \\
\hline 25 & -0.21 & 0.39 & 2.06 & 1.85 & 1.67 & 0.61 & 1.64 & 0.50 & -0.70 & 3.41 & 2.80 & -1.30 & -1.18 \\
\hline 26 & -0.16 & 0.36 & 2.06 & 1.90 & 1.70 & 0.59 & 1.68 & 0.52 & -0.65 & 3.60 & 2.89 & -1.24 & -1.11 \\
\hline 27 & -0.09 & 0.35 & 2.06 & 1.97 & 1.71 & 0.58 & 1.73 & 0.55 & -0.60 & 3.89 & 2.93 & -1.19 & -1.03 \\
\hline 28 & -0.06 & 0.34 & 2.06 & 2.00 & 1.72 & 0.56 & 1.78 & 0.58 & -0.55 & 3.99 & 2.97 & -1.10 & -0.95 \\
\hline 29 & -0.05 & 0.29 & 2.06 & 2.01 & 1.77 & 0.55 & 1.83 & 0.60 & -0.50 & 4.04 & 3.13 & -1.01 & -0.89 \\
\hline 30 & -0.04 & 0.29 & 2.06 & 2.02 & 1.77 & 0.53 & 1.88 & 0.63 & -0.46 & 4.09 & 3.15 & -0.93 & -0.81 \\
\hline 31 & -0.04 & 0.26 & 2.06 & 2.02 & 1.80 & 0.52 & 1.94 & 0.66 & -0.41 & 4.09 & 3.23 & -0.83 & -0.74 \\
\hline 32 & 0.04 & 0.24 & 2.06 & 2.10 & 1.82 & 0.50 & 2.00 & 0.69 & -0.37 & 4.39 & 3.33 & -0.77 & -0.67 \\
\hline 33 & 0.24 & 0.04 & 2.06 & 2.30 & 2.02 & 0.48 & 2.06 & 0.72 & -0.32 & 5.27 & 4.09 & -0.74 & -0.65 \\
\hline 34 & 0.26 & -0.04 & 2.06 & 2.32 & 2.10 & 0.47 & 2.13 & 0.76 & -0.28 & 5.39 & 4.40 & -0.64 & -0.58 \\
\hline 35 & 0.29 & -0.04 & 2.06 & 2.35 & 2.10 & 0.45 & 2.21 & 0.79 & -0.23 & 5.51 & 4.40 & -0.55 & -0.49 \\
\hline 36 & 0.29 & -0.05 & 2.06 & 2.35 & 2.11 & 0.44 & 2.29 & 0.83 & -0.19 & 5.53 & 4.46 & -0.45 & -0.40 \\
\hline 37 & 0.34 & -0.06 & 2.06 & 2.40 & 2.12 & 0.42 & 2.37 & 0.86 & -0.15 & 5.74 & 4.51 & -0.35 & -0.31 \\
\hline 38 & 0.35 & -0.09 & 2.06 & 2.41 & 2.15 & 0.41 & 2.46 & 0.90 & -0.10 & 5.80 & 4.62 & -0.25 & -0.22 \\
\hline 39 & 0.36 & -0.16 & 2.06 & 2.42 & 2.22 & 0.39 & 2.56 & 0.94 & -0.06 & 5.86 & 4.94 & -0.15 & -0.14 \\
\hline 40 & 0.39 & -0.21 & 2.06 & 2.45 & 2.27 & 0.38 & 2.67 & 0.98 & -0.02 & 5.98 & 5.17 & -0.05 & -0.04 \\
\hline 41 & 0.41 & -0.24 & 2.06 & 2.47 & 2.30 & 0.36 & 2.78 & 1.02 & 0.02 & 6.11 & 5.28 & 0.06 & 0.05 \\
\hline 42 & 0.44 & -0.26 & 2.06 & 2.50 & 2.32 & 0.34 & 2.91 & 1.07 & 0.07 & 6.23 & 5.40 & 0.16 & 0.15 \\
\hline 43 & 0.46 & -0.31 & 2.06 & 2.52 & 2.37 & 0.33 & 3.05 & 1.11 & 0.11 & 6.36 & 5.63 & 0.27 & 0.26 \\
\hline 44 & 0.46 & -0.31 & 2.06 & 2.52 & 2.37 & 0.31 & 3.20 & 1.16 & 0.15 & 6.36 & 5.63 & 0.38 & 0.36 \\
\hline 45 & 0.49 & -0.34 & 2.06 & 2.55 & 2.40 & 0.30 & 3.37 & 1.21 & 0.19 & 6.48 & 5.75 & 0.49 & 0.47 \\
\hline 46 & 0.54 & -0.34 & 2.06 & 2.60 & 2.40 & 0.28 & 3.56 & 1.27 & 0.24 & 6.74 & 5.75 & 0.62 & 0.57 \\
\hline 47 & 0.56 & -0.41 & 2.06 & 2.62 & 2.47 & 0.27 & 3.76 & 1.33 & 0.28 & 6.87 & 6.09 & 0.74 & 0.70 \\
\hline 48 & 0.74 & -0.46 & 2.06 & 2.80 & 2.52 & 0.25 & 4.00 & 1.39 & 0.33 & 7.82 & 6.37 & 0.91 & 0.82 \\
\hline 49 & 0.74 & -0.71 & 2.06 & 2.80 & 2.77 & 0.23 & 4.27 & 1.45 & 0.37 & 7.82 & 7.67 & 1.04 & 1.03 \\
\hline 50 & 0.91 & -0.71 & 2.06 & 2.97 & 2.77 & 0.22 & 4.57 & 1.52 & 0.42 & 8.83 & 7.69 & 1.24 & 1.16 \\
\hline 51 & 0.94 & -0.76 & 2.06 & 3.00 & 2.82 & 0.20 & 4.92 & 1.59 & 0.47 & 8.98 & 7.97 & 1.40 & 1.32 \\
\hline 52 & 0.96 & -0.76 & 2.06 & 3.02 & 2.82 & 0.19 & 5.33 & 1.67 & 0.52 & 9.13 & 7.97 & 1.56 & 1.45 \\
\hline
\end{tabular}




\begin{tabular}{|c|c|c|c|c|c|c|c|c|c|c|c|c|c|}
\hline$n$ & ${ }^{*} E$ & ${ }^{\star} E$ & $\hat{y}_{5}$ & $x^{\prime}$ & $x^{\prime \prime}$ & $1-n / N$ & $(1-n / N)^{-1}$ & $Y$ & $Y$ & $(X)^{2}$ & $(X)^{2}$ & $X^{\prime} \cdot Y$ & $X^{\prime \prime} \cdot Y$ \\
\hline 53 & 0.96 & -0.86 & 2.06 & 3.02 & 2.92 & 0.17 & 5.82 & 1.76 & 0.57 & 9.13 & 8.55 & 1.71 & 1.65 \\
\hline 54 & 0.96 & -0.86 & 2.06 & 3.02 & 2.92 & 0.16 & 6.40 & 1.86 & 0.62 & 9.13 & 8.55 & 1.87 & 1.81 \\
\hline 55 & 0.99 & -0.86 & 2.06 & 3.05 & 2.92 & 0.14 & 7.11 & 1.96 & 0.67 & 9.28 & 8.55 & 2.05 & 1.97 \\
\hline 56 & 0.99 & -0.91 & 2.06 & 3.05 & 2.97 & 0.13 & 8.00 & 2.08 & 0.73 & 9.28 & 8.84 & 2.23 & 2.18 \\
\hline 57 & 1.04 & -0.91 & 2.06 & 3.10 & 2.97 & 0.11 & 9.14 & 2.21 & 0.79 & 9.59 & 8.84 & 2.46 & 2.36 \\
\hline 58 & 1.04 & -0.94 & 2.06 & 3.10 & 3.00 & 0.09 & 10.67 & 2.37 & 0.86 & 9.59 & 8.99 & 2.67 & 2.58 \\
\hline 59 & 1.06 & -0.94 & 2.06 & 3.12 & 3.00 & 0.08 & 12.80 & 2.55 & 0.94 & 9.74 & 8.99 & 2.92 & 2.81 \\
\hline 60 & 1.06 & -0.94 & 2.06 & 3.12 & 3.00 & 0.06 & 16.00 & 2.77 & 1.02 & 9.74 & 8.99 & 3.18 & 3.06 \\
\hline 61 & 1.09 & -0.99 & 2.06 & 3.15 & 3.05 & 0.05 & 21.33 & 3.06 & 1.12 & 9.90 & 9.29 & 3.52 & 3.41 \\
\hline 62 & 1.09 & -1.00 & 2.06 & 3.15 & 3.06 & 0.03 & 32.00 & 3.47 & 1.24 & 9.93 & 9.37 & 3.92 & 3.80 \\
\hline 63 & 1.14 & -1.04 & 2.06 & 3.20 & 3.10 & 0.02 & 64.00 & 4.16 & 1.43 & 10.22 & 9.60 & 4.56 & 4.42 \\
\hline 64 & 1.15 & -1.14 & 2.06 & 3.21 & 3.20 & 0.00 & \#DIV/0! & \#DIV/0! & 2 & 10.30 & 10.23 & 6.42 & 6.40 \\
\hline$\sum$ & & & & 136.9 & 126.8 & & & & -32.8 & 325.8 & 284.2 & -17.3 & -12.7 \\
\hline
\end{tabular}

\section{Testing result intrepretation}

On the basis of the obtained data it follows that:

$\hat{y}=b_{0}+b_{1} x_{1}+b_{2} x_{2}+b_{3} x_{3}+b_{4} x_{4}+b_{5} x_{5}+b_{6} x_{6}+$ $+b_{7} x_{7}+b_{24} x_{2} x_{4}+b_{23} x_{2} x_{3}+b_{16} x_{1} x_{6}+b_{26} x_{2} x_{6}+$ $+b_{45} x_{4} x_{5}+b_{35} x_{3} x_{5}+b_{56} x_{5} x_{6}$.

$\hat{y}=3.39-0.93 x_{1}-0.59 x_{2}-0.73 x_{3}-0.98 x_{4}-0.23 x_{5}$ $+0.84 x_{6}+0.5 x_{7}+0.47 x_{2} x_{4}-0.07 x_{2} x_{3}-0.27 x_{1} x_{6}-$

$0.31 x_{2} x_{6}+0.1 x_{4} x_{5}+0.48 x_{3} x_{5}-0.1 x_{5} x_{6}$

On the basis of Table 4 and the method of least squares, and after furder processing of the results, as describe in literature (Koldžić, 1999), using transformation equitation (2) (Koldžić, 1999), (Stanić, 1990),

$$
x=\frac{X-X_{o}}{w}, X_{o}=\frac{X_{g}+X_{d}}{2}
$$

it is obtained:

$\alpha=1.6$

$\ln \beta=9.48-0.66 M_{r}-1.3 \sigma_{r}-1.1 M_{a}-2.44 \sigma_{a}+$ $0.39 k+4.5 \eta_{c}+0.33 D-0.84 \sigma_{r} \sigma_{a}-0.1 \sigma_{r} M_{a}-$ $0.54 M_{r} \eta_{c}-0.83 \sigma_{r} \eta_{c}-0.07 \sigma_{a} k+0.24 M_{a} k-$ $0.1 k \eta_{c}$

The data from Table 4 are entered into the probability chart in Figure 1 and the line, which corresponds to these data.

The obtained reliability indicators relate to the whole defined multi-factorial space, from minimum to maximum values of influential factors, on the basis of wich can, therefore, reliability in each point of the experimental space be calculated on the basis of the expression:

$$
R_{(r)}=e^{-(r / \beta)^{\alpha}}
$$

where - $R(r)$ reliability depends on the $r$ number of revolutions.

For example, if it has been determined that a roller bearing will operate in the operating conditions where

$$
\begin{gathered}
M_{r}=2.4 ; \quad \sigma_{r}=1 ; \quad M_{a}=1.1 ; \quad \sigma_{a}=1 ; \\
\quad k=3.5 ; \quad \eta=0.8
\end{gathered}
$$

and for the limit value of diagnostic parameter $D=6$, so that for the given operational conditions the values of $\alpha=1.6$ and $\beta=963$ are obtained, reliability for some numbers of revolution is as given in Table 5 .

Table 5 Reliability and number of revolucions

\begin{tabular}{|c|c|}
\hline $\begin{array}{c}\text { Reliability } \\
R(r)[\%]\end{array}$ & $\begin{array}{c}\text { Number of revolutions } \\
r\end{array}$ \\
\hline $90(0.90)$ & $2280 \times 10^{6}$ \\
\hline $95(0.95)$ & $1505 \times 10^{6}$ \\
\hline $99(0.99)$ & $540 \times 10^{6}$ \\
\hline
\end{tabular}

On the basis of the (1) functional dependence, the functional dependence of diagnostical parameter on thenumber of revolutions can be arrived at. Namely, for any known operation conditions the (1) expression looks like this:

$$
\hat{y}=\ln _{\hat{r}}=A+0.5 x_{7}
$$

and (Draper \& Smith, 1966), (Koldžić, 1999): 
MEST Journal Vol.1 No.1 pp. 37 - 45

$$
y=\ln r=A+0.5 x_{7}+\varepsilon
$$

where $A$ represent constant value, while $\varepsilon$ is a random quantity for the observed population.

After entering of transformation equation (2) into the (5) expressions, the results are obtained in the form of:

$D=C+3 \ln r$

This is obviously the case of a logarithmic random function, since $C$ is a random quantity for one population and for one level of the diagnostical parameter. This random function can be approximately replaced, within the scope of the diagnostical parameter from $D_{\min }=3$ to $D_{\max }=6$ by a linear random function.

The obtained data regarding the diagnostical parameter enable forming of the most promising and most economical models of technical systems maintenance - the models of preventive maintenance according to condition. The experimental results interpreted in this way, from the point of view of diagnostic parameter enable predicting of operating time to failure (of the number of revolutions till failure in this example), on the basis of only one check-up of the state. Namely, on the basis of the results of the mentioned check-up of the state, the $C$ quantity in the (6) expression can be obtained, so that this random function after one diagnostic becomes deterministic.

\section{CONCLUSION}

Briefly stated, the advantages of this method are as follows:

1) Enormous decrease of the number of experiments, and thereby of the costs of reliability testing in a multi-factorial space. As can be seen on the Table 2, the experiments have been conducted four times in each point of the plan, on four elements (roller bearings). Since, according to the testing plan matrix, the experiments are conducted at 16 points, the total number of elements in the sample is $4 \times 16=64$. The number of analytically reached data relevant to reliability in defined multi-factorial space, in this example is $64 \times 16=1024$.

2) Interpretation of testing results is enabled in the form of Weibull distribution law in the whole defined multi-factorial space;

3) The function of the diagnostical parameter change in time is attained within the desired scope of the parameter;

4) This method does not comprise any casual improvisations so that results are absolutely accurate.

\section{Works Cited}

Draper, N., \& Smith, H. (1966). Applied Regression Analysis. New York: John Wiley \& Sons.

Koldžić, M. (1999). The Method of Accelerated Multi-Factor Testing of Reliability of Mechanical System Elements. Belgrade: University Belgrade, Faculty of Mechanical Engineering.

Stanić, J. (1990). The Method of Engineering Measuring. Belgrade: University Belgrade, Faculty of Mechanical Engineering .

Received for publication: $\quad 25.09 .2012$

Accepted for publication: $\quad$ 16.11.2012 
Arsenić Ž. Multifactorial testing of bearing reliability MEST Journal Vol.1 No.1 pp. 37-45 


\section{How to cite this article?}

Style - APA Sixth Edition:

Arsenić, Ž. (2013, 01 15). Multifactorial testing of bearing reliability. (Z. Čekerevac, Ed.) MEST Journal, 1(1), 37-45. Retrieved from www.meste.org/mest/MEST_1_2013/_04.pdf. DOI 10.12709/mest.01.01.01.04.pdf

Style - Chicago Fifteenth Edition:

Arsenić, Živan. "Multifactorial testing of bearing reliability." Edited by Zoran Čekerevac. MEST Journal (MESTE NGO) 1, no. 1 (01 2013): 37-45.

Style - GOST Name Sort:

Arsenić Živan Multifactorial testing of bearing reliability [Journal] = Multifactorial testing of bearing reliability // MEST Journal / ed. Čekerevac Zoran. - Belgrade - Toronto : MESTE NGO, 01 15, 2013. - 1 : Vol. 1. - pp. 37-45.

Style - Harvard Anglia:

Arsenić, Ž., 2013. Multifactorial testing of bearing reliability. MEST Journal, 15 01, 1(1), pp. $37-45$.

Style - ISO 690 Numerical Reference:

Multifactorial testing of bearing reliability. Arsenić, Živan. [ed.] Zoran Čekerevac. 1, Belgrade - Toronto : MESTE NGO, 01 15, 2013, MEST Journal, Vol. 1, pp. 37-45. 